\title{
Stability of West Nile virus in lysis solution and its implication on One Health
}

\author{
Jesse J. C. Erandio ${ }^{1,2}$, Mustapha Debboun ${ }^{2}$ \\ ${ }^{1}$ California Department of Public Health, Pacific Architects and Engineers, Richmond, CA 94804, USA. \\ ${ }^{2}$ Delta Mosquito and Vector Control District, Lourenco Laboratory, Visalia, CA 93291, USA.
}

Correspondence to: Dr. Mustapha Debboun, Delta Mosquito and Vector Control District, Lourenco Laboratory, 1737 W. Houston Ave, Visalia, CA 93291, USA. E-mail: mdebboun@deltamvcd.org

How to cite this article: Erandio JJC, Debboun M. Stability of West Nile virus in lysis solution and its implication on One Health. One Health Implement Res 2021;1:56-65. https://dx.doi.org/10.20517/ohir.2021.06

Received: 27 May 2021 First Decision: 8 Oct 2021 Revised: 18 Oct 2021 Accepted: 10 Nov 2021 Published: 25 Nov 2021

Academic Editor: Jorg Heukelbach Copy Editor: Yue-Yue Zhang Production Editor: Yue-Yue Zhang

\begin{abstract}
Aim: The stability of nucleic acids varies based on storage conditions and plays an important role in achieving optimal laboratory operations. This study was conducted to implement One Health into vector surveillance and enhance our understanding of the stability of West Nile virus (WNV) positive mosquito homogenate in the Ambion ${ }^{\circledR}$ MagMAX ${ }^{\mathrm{TM}}$ lysis/binding solution to optimize laboratory operations.

Methods: WNV positive mosquito pools used for this study were collected within the Delta Mosquito and Vector Control District boundaries in 2017. Homogenates were combined and aliquoted into five sample sets. Each sample set was stored in the corresponding storage: the $-20^{\circ} \mathrm{C}$ freezer, $+4{ }^{\circ} \mathrm{C}$ refrigerator, at $+23^{\circ} \mathrm{C}$ room temperature environment, and two sets were stored in the $-80^{\circ} \mathrm{C}$ freezer where one set underwent four freeze-thaw cycles every week for 18 weeks. Each sample was processed through ribonucleic acid extraction and real-time quantitative reverse transcription-polymerase chain reactions (RT-qPCR), four days a week, for 18 weeks.
\end{abstract}

Results: On the 18th week of the study, WNV remained detectable through RT-qPCR under all five storage conditions, including 64 freeze-thaw cycles. A statistically significant difference in weekly mean cyclic threshold values was found between the $-80^{\circ} \mathrm{C}$ and freeze-thaw sample sets.

Conclusion: By using the Ambion ${ }^{\circledR}$ MagMAX ${ }^{\mathrm{TM}}$ lysis/binding solution, viral samples can be stored under nonoptimal conditions for at least four months. With climatic changes occurring, vector-borne disease transmission increases. Therefore, increasing the need for global vector control and surveillance, implementation of One Health 
strategies will improve public and animal health through optimized vector-borne disease control, surveillance, and prevention.

Keywords: West Nile virus, storage temperature, One Health, freeze-thaw, lysis/binding solution, cyclic threshold, reagent, extraction

\section{INTRODUCTION}

West Nile virus (WNV), St. Louis Encephalitis virus (SLEV), Western Equine Encephalitis virus (WEEV), as well as other vector-borne viruses, are important pathogens that mosquito and vector control districts monitor to support public health and engage in One Health. As climate change takes effect across the globe, the geographical distribution of invasive vectors and vector-borne pathogens has become widespread and prompted an increase in arthropod-borne virus (arbovirus) surveillance ${ }^{[1]}$. Mosquito and vector control districts across the United States work closely with other agencies and state health departments. Close communication with public health departments analyzes human and animal cases for vector-borne diseases, such as WNV. Collaboration with other agencies helps develop new surveillance and control methods. On the other hand, communicating with environmental and pesticide regulation departments ensure safety guidelines are met prior to chemical treatments. As WNV, SLEV, and WEEV have become established in California, the transmission cycle of the viruses plays an essential role in providing optimal surveillance and control efforts ${ }^{[2]}$. It is known that birds serve as reservoir hosts for WNV, SLEV, and WEEV, while humans serve as dead-end hosts. Additionally, horses also serve as dead-end hosts, but only for WNV and WEEV. By understanding the transmission cycles of these three established mosquito-borne viruses in California, close communication with public health, regulatory, and environmental departments helps mosquito and vector control districts provide the best health outcomes through One Health collaborations ${ }^{[3]}$. Jointly, vector control agencies develop One Health strategies, such as optimizing arbovirus surveillance to anticipate and mitigate outbreaks in public health.

As One Health encompasses the assessment of human, animal, and environmental factors, establishing vector control laboratories will allow public health departments to analyze the presence, distribution, and health outcomes of vector-borne diseases. Delta Mosquito and Vector Control District, located in Visalia, California, was established in 1922 and has grown and evolved to an important One Health agency performing vector and vector-borne disease surveillance [Figure 1]. Since the geographical expansion of WNV throughout California in 2004, mosquito surveillance has increased across the state $\mathrm{e}^{[4]}$. The increase in state vector surveillance efforts for WNV includes trapping for gravid female Culex spp. mosquitoes, chemical treatment of large stagnant bodies of water, the use of mosquitofish, Gambusia affinis for biological control, and performing real-time quantitative polymerase chain reactions (RT-qPCR) to detect WNV, as well as SLEV and WEEV ${ }^{[5]}$. In 2011, the Lourenco Laboratory at Delta Mosquito and Vector Control District was built to perform testing for mosquito-borne diseases, primarily WNV, SLEV, and WEEV. Since the establishment of the Lourenco Laboratory, it has added and implemented a rigorous testing method of ribonucleic acid (RNA) extraction and RT-qPCR for WNV, SLEV, WEEV, Chikungunya virus, Dengue virus, and Zika virus. In addition, the District has recently implemented surveillance efforts for all vectors present within its jurisdiction, such as ticks for Lyme disease and triatomines for Chagas disease. This way, the increase in surveillance efforts encourages communication between local communities, health departments, and environmental agencies in order to optimize integrative vector management and One Health across multiple disciplines. By establishing a robust testing method, it was important to understand the shelf life of the chemicals being used to optimize arboviral testing. Therefore, this study closely examined the Ambion MagMAX ${ }^{\mathrm{mox}}$ lysis binding solution concentrate (Cat. No. AM8500, Thermo Fisher Scientific, Lithuania) and its effects on the quality of WNV in positive mosquito samples. 




Figure 1. Tulare County is located in central California and is outlined in red (left). Major cities within the Delta Mosquito and Vector Control District boundaries include, but are not limited to, Cutler (1), Dinuba (2), Exeter (3), Farmersville (4), Goshen (5), Orosi (6), Visalia (7), and Woodlake (8) which are outlined in blue (right).

When conducting and optimizing arboviral testing, storage conditions play a crucial role in understanding the quality of chemical reagents and laboratory samples. The stability of viral nucleic acids in buffer solutions can vary based on storage conditions $s^{[6-9]}$. When cells, RNA, DNA, protein, bacteria, or viruses are stored in ultra-low temperature storages, molecular components continue to degrade as biochemical processes and pathways continue to function at a slower rate ${ }^{[10]}$. In this case, knowledge about the shelf life is essential to maintaining the quality and integrity of specimens and chemical reagents. The goal of this study was to enhance our understanding of the stability of WNV positive mosquito homogenate in the Ambion ${ }^{\circ}$ MagMAX ${ }^{\mathrm{mm}}$ lysis/binding solution based on five storage conditions: storing samples in the $-80{ }^{\circ} \mathrm{C}$ and $-20{ }^{\circ} \mathrm{C}$ freezers, $+4{ }^{\circ} \mathrm{C}$ refrigerator, at $+23{ }^{\circ} \mathrm{C}$ room temperature environment, and undergoing multiple numbers of freeze-thaw cycles. The analysis and results of the preliminary study were presented and published as a brief proceeding at the 88th Annual Conference of the Mosquito and Vector Control Association of California ${ }^{[11]}$. In this paper, the expansion and the molecular components of the study were further analyzed in detail, and the connection between the study and One Health was expounded on. Moreover, this study allowed the laboratory staff to provide accurate arboviral test results, enhance its support to improve public health, and optimize laboratory capabilities by implementing a One Health strategy.

\section{METHODS}

Twenty-seven WNV positive mosquito pools that were collected within the Delta Mosquito and Vector Control District boundaries from 2017 were combined into one $50 \mathrm{~mL}$ conical tube. Each mosquito pool had $500 \mu \mathrm{L}$ of retrievable homogenate that is in the Ambion ${ }^{\circ}$ MagMAX $^{\mathrm{mm}}$ lysis/binding solution, and a total of $13.5 \mathrm{~mL}$ of homogenate was accumulated. The combined homogenate from the $50 \mathrm{~mL}$ conical tube was vortexed for $1 \mathrm{~min}$, centrifuged for $5 \mathrm{~min}$ with a relative centrifugal force of $1276 \mathrm{~g}$, and aliquoted into five sets of 26 tubes. Each set was stored in an enclosed box and placed in the corresponding storage. One sample set was stored in the $-20^{\circ} \mathrm{C}$ freezer, $+4{ }^{\circ} \mathrm{C}$ refrigerator, at $+23{ }^{\circ} \mathrm{C}$ room temperature environment, and two sets were stored in the $-80^{\circ} \mathrm{C}$ freezer where one set underwent four freeze-thaw (F-T) cycles every week for 18 weeks. 
Viral RNA extraction and RT-qPCR protocols used for this study followed the same procedures as the Delta Mosquito and Vector Control District multiplex RT-qPCR for WNV, SLEV, and WEEV. Aliquoted samples were extracted using the MagMAX ${ }^{\mathrm{m}}$ Express Magnetic Particle Processor (Thermo Fisher Scientific, Waltham, Massachusetts, USA) with the MagMAX ${ }^{\mathrm{m}}$-96 Viral RNA Isolation kit (Cat. No. AMB18365, Applied Biosystems, Foster City, California, USA). The RT-qPCR was performed using the TaqMan ${ }^{\text {mi }}$ Fast Virus 1-Step Master Mix (Cat. No. 4444434, Applied Biosystems, Foster City, California, USA). A multiplex primer-probe set, targeting the envelope protein, was used for this project that simultaneously detects WNV, SLEV, and WEEV [Table 1]. Each component for the primer-probe set was optimized specifically for Delta Mosquito and Vector Control District [Table 2]. Formulation of this recipe was an adaptation from the Davis Arbovirus Research and Training Laboratory, located in the University of California, Davis, where WNV, SLEV, and WEEV primer-probe sets were combined to create a multiplex RT-qPCR test for mosquito and dead bird samples across California ${ }^{[12]}$. The same primer-probe sets were used to perform Delta Mosquito and Vector Control District's routine arboviral testing of mosquito pools alongside this study.

All samples were tested on the first week and then regularly on the 13th week because the same study was conducted in 2018, but it only lasted up to week 13 due to budgetary constraints, where WNV remained detectable. Additionally, storage temperatures were measured three times daily and were averaged weekly. Weekly mean cyclic threshold values were recorded and used to calculate cyclic threshold differences and variations of each sample set ${ }^{[13-16]}$. Weekly mean cyclic threshold values for each sample set were also compared to the $-80^{\circ} \mathrm{C}$ sample set using multiple paired $t$-tests since storing samples in the $-80{ }^{\circ} \mathrm{C}$ freezer is a standard storage condition.

\section{RESULTS}

West Nile virus positive mosquito homogenate remained detectable under all five storage conditions for 18 weeks and $64 \mathrm{~F}$-T cycles. As for storage temperatures, all, except the $-80{ }^{\circ} \mathrm{C}$ freezer, were three degrees lower than expected [Figure 2]. Compared to the initial mean cyclic threshold reading shown in Table 3, the values dropped an average of 3.5 values [Figure 3]. However, weekly mean cyclic threshold differences did not differ more than two values from week 13 to 18 .

As WNV remained detectable in mosquito homogenate for 18 weeks and 64 freeze-thaw cycles, multiple paired $t$-tests on the weekly mean cyclic threshold values between each sample set and the $-80^{\circ} \mathrm{C}$ sample set showed a significant difference between $-80^{\circ} \mathrm{C}$ and F-T sample sets, with $P=0.003$. Notable variations in cyclic threshold values were detected in both the $-20{ }^{\circ} \mathrm{C}$ and $+23{ }^{\circ} \mathrm{C}$ sample sets along with high cyclic threshold readings [Figure 4]. The $+4{ }^{\circ} \mathrm{C}$ sample set had the largest drop in cyclic threshold values while consistently maintaining lower cyclic threshold values throughout the study.

\section{DISCUSSION}

This study showed that WNV remained detectable in the Ambion ${ }^{\circ} \mathrm{MagMAX}^{\mathrm{ND}}$ lysis/binding solution for 18 weeks under five storage conditions. In a similar RNA stability study, lysis buffers were found to minimize RNA degradation, such as for influenza virus RNA, for samples being stored in the $-20^{\circ} \mathrm{C}$ freezer and $+4{ }^{\circ} \mathrm{C}$ refrigerator, but not at room temperature ${ }^{[17]}$. In this case, previous studies showed limited findings in terms of the stability of RNA, using lysis buffers to directly preserve samples, and correlating these elements with storage conditions. Although WNV was amplified through RT-qPCR in all five sample sets, a large drop in cyclic threshold values was observed from week one to week 13. The remaining extraction reagents from the previous year were used for the initial testing on the first week of the study, whereas new reagents were used on week 13. This resulted in a large drop in cyclic threshold values for all samples tested. The initial 
Table 1. Sequences of the primers and probes used to simultaneously detect West Nile virus (WNV), Saint Louis encephalitis virus (SLEV), and Western Equine Encephalitis virus (WEEV) through RT-qPCR at Delta Mosquito and Vector Control District

\begin{tabular}{ll}
\hline & \multicolumn{1}{c}{ Primer and probe sequences } \\
\hline Primers and probes & Sequences \\
\hline SLEV forward primer & 5' d CTGGCTGTCGGAGGGATTCT 3' \\
SLEV probe & 5' d 5-TAMRA-TCTGGCGACCAGCGTGCAAGCCG-BHQ-2 3' \\
SLEV reverse primer & 5' d TAGGTCAATTGCACATCCCG 3' \\
WEEV forward primer & 5' d AGGTAAACTGCACATTCCATTCC 3' \\
WEEV probe & 5' d quasar 670-CCGACAGTCTGCCCGGTTCCG-BHQ-3 3' \\
WEEV reverse primer & 5' d TTCGTGACTGTAGGCGTGTGA 3' \\
WNV forward primer & 5' d TCAGCGATCTCTCCACCAAAG 3' \\
WNV probe & 5' d FAM-TGCCCGACCATGGGAGAAGCT-BHQ-1 3' \\
WNV reverse primer & 5' d GGGTCAGCACGTTTGTCATTG 3' \\
\hline
\end{tabular}

Table 2. Primer and probe concentrations (nM) used per reaction

\begin{tabular}{ll}
\hline & Primer and probe concentrations per reaction \\
\hline Primers and probes & Concentration (nM) \\
\hline St. Louis Encephalitis virus forward primer & 200 \\
St. Louis Encephalitis virus probe & 125 \\
St. Louis Encephalitis virus reverse primer & 200 \\
Western Equine Encephalitis virus forward primer & 100 \\
Western Equine Encephalitis virus probe & 83 \\
Western Equine Encephalitis virus reverse primer & 100 \\
West Nile virus forward primer & 100 \\
West Nile virus probe & 42 \\
West Nile virus reverse primer & 100 \\
\hline
\end{tabular}

Table 3. Initial mean cyclic threshold values took on the first week of the study. These values were used to calculate the weekly mean cyclic threshold difference of each sample set

\begin{tabular}{ll}
\hline & Initial mean cyclic threshold values (week 1) \\
\hline Storage condition & Cyclic threshold value \\
\hline$-80^{\circ} \mathrm{C}$ & 27.7 \\
Freeze-thaw & 26.9 \\
$-20^{\circ} \mathrm{C}$ & 28.7 \\
$+4{ }^{\circ} \mathrm{C}$ & 28.9 \\
$+23^{\circ} \mathrm{C}$ & 28.4 \\
\hline
\end{tabular}

extraction reagents were prepared for four months before they were used for this study. The large drop in cyclic threshold values could be due to the evaporation of isopropanol in the wash solution one and the lysis binding solution concentrate, with carrier RNA that is prepared in bulk, or the evaporation of ethanol in the wash solution two. All three reagents were included in the MagMAX ${ }^{\mathrm{m}}$-96 Viral Isolation Kit. Evaporation of these alcohols could prevent nucleic acids from precipitating in the lysis binding solution, and the alcohols could also prevent wash buffer solutions from removing residual binding solutions as stated on the MagMAX $^{\mathrm{m}}-96 \mathrm{AI} / \mathrm{ND}$ Viral RNA Isolation Kit instruction manual (Cat. No. AM1929, Applied Biosystem, Foster City, California, USA). Since the RNA extractions of mosquito pools were processed through a magnetic bead-based RNA purification system in this study, inadequate washing of contaminants and residual binding solution could decrease the quantity and quality of purified RNA. 


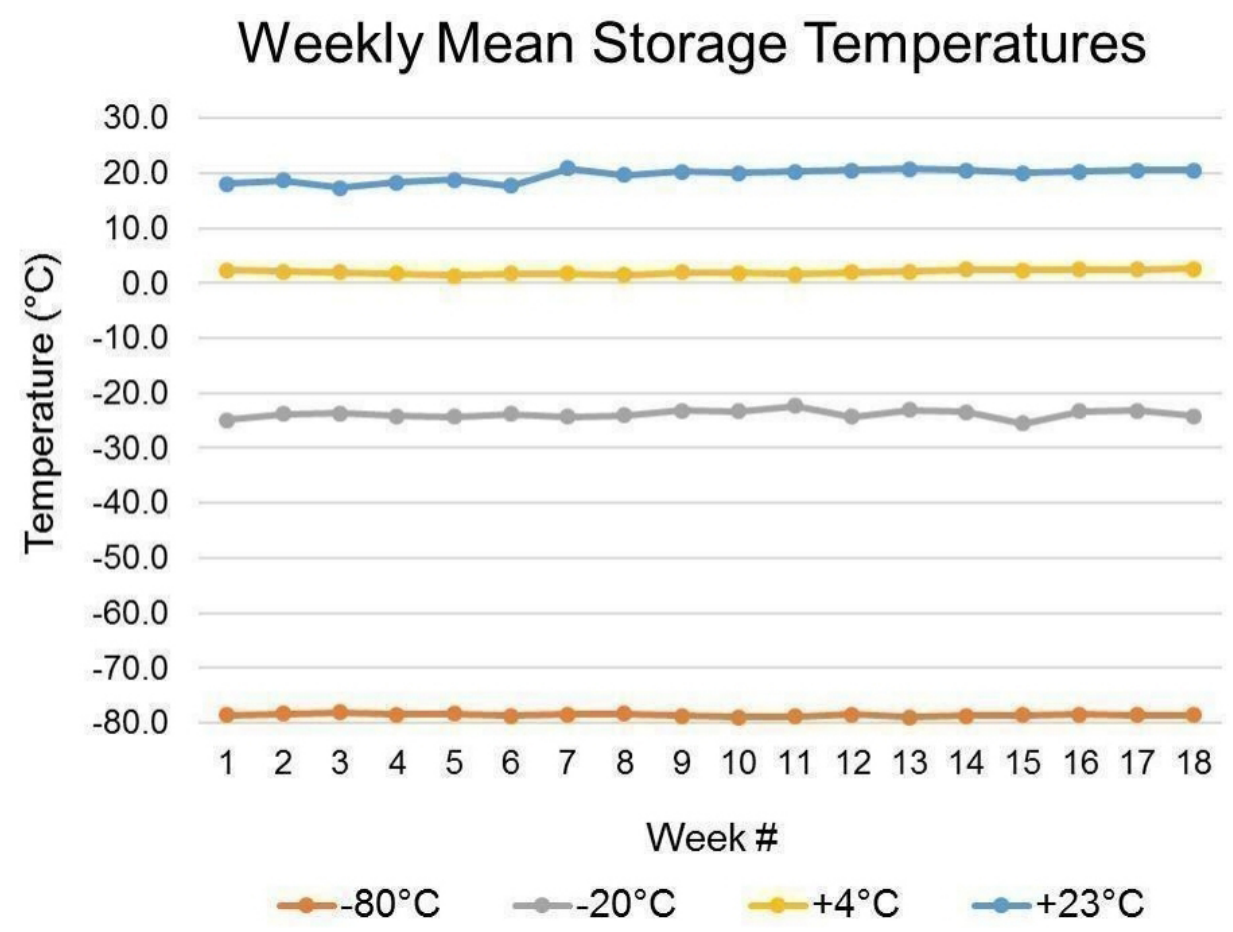

Figure 2. Weekly mean storage temperatures. All recorded temperatures, except for the $-80^{\circ} \mathrm{C}$ freezer, were three degrees lower than expected.

Weekly Mean Cyclic Threshold Difference (Weeks 13-18)

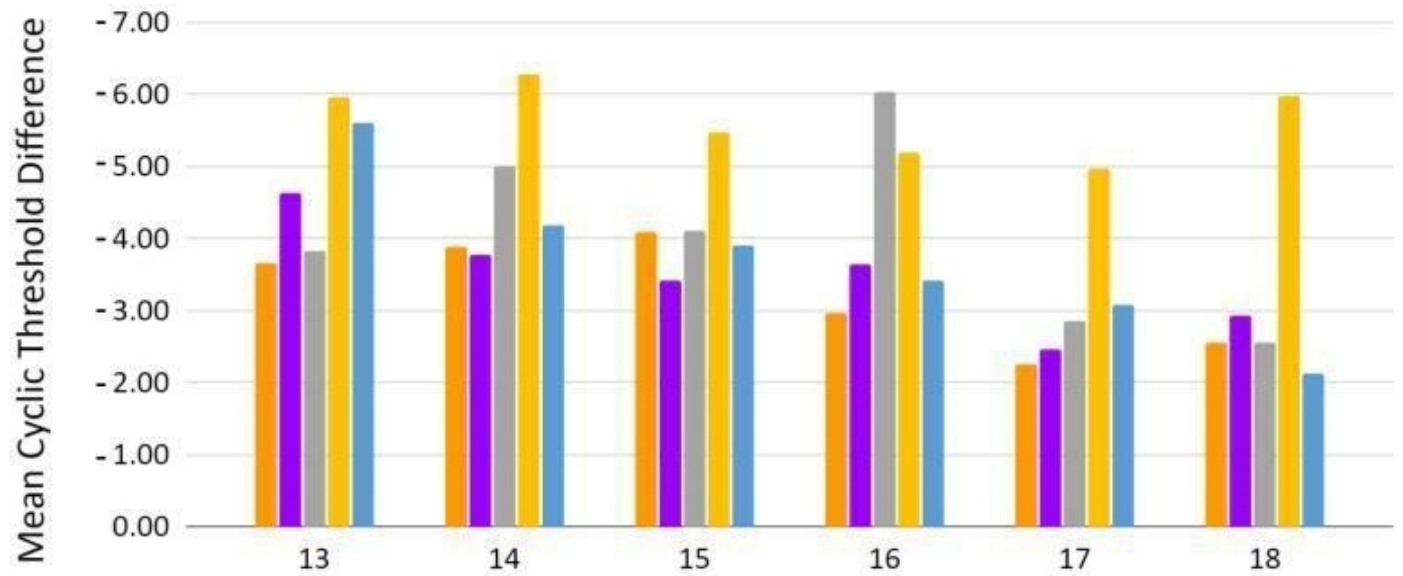

Week \#

$=-80^{\circ} \mathrm{C}=\mathrm{F}-\mathrm{T}^{*}=-20^{\circ} \mathrm{C}=+4^{\circ} \mathrm{C}=+23^{\circ} \mathrm{C}$

Figure 3. Weekly mean cyclic threshold difference of each sample set. The difference was calculated by taking the mean cyclic threshold value of a sample set, on a specific week, minus the initial mean cyclic threshold value for the corresponding sample set. The cyclic threshold values dropped an average of 3.5 values from their initial cyclic threshold value. *Denotes freeze-thaw.

Although extraction reagents were refreshed on week 13 of the study and caused a large drop in cyclic threshold values, notable variations in cyclic threshold values were observed in all sample sets except for the 


\section{Mean \pm Standard Deviation Cyclic Threshold Variation}

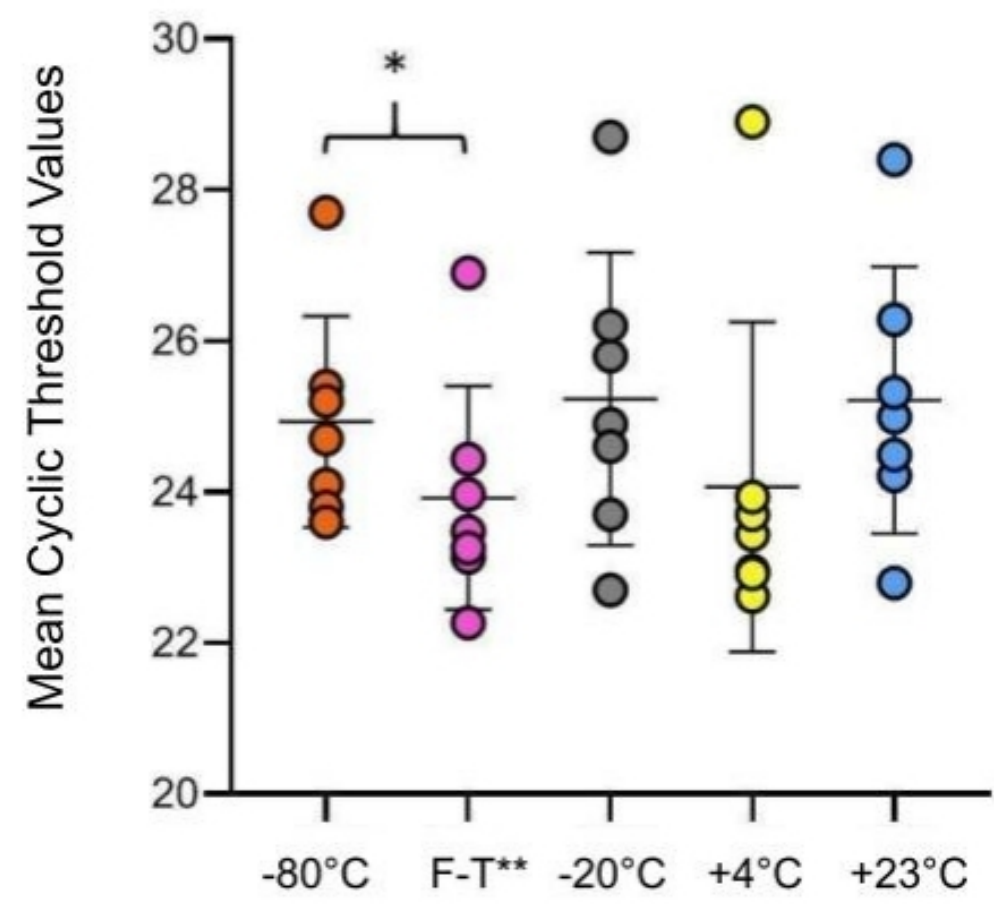

\section{Storage Condition}

Figure 4. Weekly mean cyclic threshold variation for each sample set. The highest value readings were from the first week of the study. ${ }^{\star}$ Denotes significance by $t$-test at 0.05 probability level; ${ }^{\star *}$ Denotes freeze-thaw.

$+4{ }^{\circ} \mathrm{C}$ sample set. Compared to the $-80{ }^{\circ} \mathrm{C}$ sample set, the F-T sample set initially had a lower cyclic threshold value reading along with moderate fluctuations in cyclic threshold values throughout the study [Figure 4]. The F-T sample set consisted of variations in cyclic threshold values because the sample set cumulatively underwent multiple freeze-thaw cycles within 18 weeks, therefore, degrading detectable viral RNA in the homogenate $e^{[18]}$. As for the $-20{ }^{\circ} \mathrm{C}$ and $+23^{\circ} \mathrm{C}$ sample sets, variation and high cyclic threshold values were observed. These conditions were expected for the $+23{ }^{\circ} \mathrm{C}$ sample set as these samples were being stored in an environment with elevated temperatures. As for the $-20{ }^{\circ} \mathrm{C}$ sample set, they were difficult to handle because the homogenate was viscous once fully thawed. Even though the $+4{ }^{\circ} \mathrm{C}$ sample set had the largest drop in cyclic threshold value from its initial reading, it had consistently lower cyclic threshold values. The $+4{ }^{\circ} \mathrm{C}$ sample set remained in the same liquid state throughout the study while being stored in a chilled environment. Variations in cyclic threshold values could have been affected by several factors, such as large amounts of RNase introduced to the samples when multiple mosquito pools were combined or the quality of buffer solutions.

As experimental results indicated that WNV remained detectable in all five storage conditions after 18 weeks, arboviral testing with the Ambion ${ }^{\odot} \mathrm{MagMAX}^{\mathrm{in}}$ lysis/binding solution concentrate allows storage of samples under non-optimal conditions. Without the need for ultra-low freezers to test mosquito samples for arbovirus, the requirements of vector control laboratories can be lessened and allow other laboratories to perform arbovirus testing. However, with recent extreme climate changes, California is experiencing longer 
periods of warmth throughout the year, which prolongs the mosquito season. With prolonged mosquito seasons, the risks of vector-borne disease transmission are increased. It is a crucial component for mosquito and vector control districts to understand the limitations of the integrity of nucleic acids in mosquito samples and potential solutions, such as using the Ambion ${ }^{\bullet}$ MagMAX $^{\mathrm{m}}$ lysis/binding solution to aid in the retention of detectable viral RNA and optimize their surveillance efforts. Therefore, it is essential that other mosquito and vector control laboratories are able to test their own mosquito collections in order to improve arboviral surveillance efforts and prevent potential human, as well as animal disease cases from occurring.

As we examined the effects of storage conditions on the stability of WNV positive mosquito samples, we began to understand the logistical impact of the experiment towards arboviral surveillance protocols and One Health strategies. As a significant global One Health concern, vector-borne diseases represent about $17 \%$ of all communicable diseases that affect human and animal health, according to the World Health Organization. By incorporating a One Health approach into vector surveillance and control, we can strengthen global vector-borne disease surveillance programs. Using the findings of this study in conjunction with One Health, human and animal health can be improved by allowing laboratories to establish arboviral testing under non-optimal storage conditions with the appropriate chemical reagents. The findings of this study also make RT-qPCR testing methods more accessible and cost-efficient for local health departments, veterinary offices, and environmental departments by requiring the use of suboptimal storage containers. This way, more entities can be involved in vector-borne disease surveillance and create a multidisciplinary approach to improve the health of animals and humans, such as reducing wildlife and domestic animal interaction, providing proper healthcare for both humans and animals, or encouraging local communities to be a part of the surveillance efforts ${ }^{[19]}$. In addition, many researchers have analyzed the impact of climatic changes on vector-borne disease transmission, such as malaria, using evidence-based models to assess current predictions and responses ${ }^{[20]}$. With the findings of this study, the effects of temperature storage can be included in evidence-based models and allow us to advance our understanding of vector-borne diseases by associating environmental elements from the field and laboratory.

In conclusion, as WNV remained detectable in mosquito homogenate in the Ambion ${ }^{\circledR}$ MagMAX $^{\mathrm{m}}$ lysis/binding solution for 18 weeks under five storage conditions, this study can be applied to vector-borne surveillance programs that do not have an ultra-low temperature freezer readily available and be able to manage vector-borne disease positive samples with ample time. Future work on virus titer measurements, comparing buffer solution stability under different storage conditions, and replicating this study on other viruses will help provide a better understanding of viral degradation ${ }^{[21-25]}$. If a laboratory does not have access to ultra-low temperature freezers or is experiencing a freezer breakdown, viral samples can be stored under non-optimal conditions for at least four months. With global climatic changes and technological advances, the length of time of transmission for arboviruses has increased as vectors have become widespread. By using the findings of this study and implementing One Health approach, other public health and vector-borne disease laboratories will be able to enhance their efforts to conduct arboviral surveillance and improve public and animal health.

\section{DECLARATIONS}

\section{Acknowledgement}

We thank the Delta Mosquito and Vector Control District staff, particularly the biologists Crystal Grippin and Mark Nakata, and Scientific Program Manager Mir Bear-Johnson, who helped in all aspects of this study during the time the study was conducted in 2018 and 2019. 


\section{Authors' contributions}

Conceptualization: Erandio JJC, Debboun M

Data Analysis, investigation, methodology: Erandio JJC

Resources: Erandio JJC, Debboun M

Writing: Erandio JJC, Debboun M

\section{Availability of data and materials}

Not applicable.

\section{Financial support and sponsorship}

None.

\section{Conflicts of interest}

Both authors declared that there are no conflicts of interest.

\section{Ethical approval and consent to participate}

Not applicable.

\section{Consent for publication}

Not applicable.

\section{Copyright}

(c) The Author(s) 2021.

\section{REFERENCES}

1. Caminade C, McIntyre KM, Jones AE. Impact of recent and future climate change on vector-borne diseases. Ann $N$ Y Acad Sci 2019;1436:157-73. DOI PubMed PMC

2. Diaz A, Coffey LL, Burkett-Cadena N, Day JF. Reemergence of St. Louis encephalitis virus in the Americas. Emerg Infect Dis 2018:24. DOI PubMed PMC

3. Faburay B. The case for a 'one health' approach to combating vector-borne diseases. Infect Ecol Epidemiol 2015;5:28132. DOI PubMed PMC

4. Hom A, Marcus L, Kramer VL, et al. Surveillance for mosquito-borne encephalitis virus activity and human disease, including West Nile virus in California. Proceedings and papers of the 73rd annual conference of the Mosquito and Vector Control Association of California; 2005 Oct; Sacramento, California. California: Mosquito and Vector Control Association of California, 2005.

5. California Mosquito-borne virus surveillance and response plan. Response plans and guidelines. Available from: https://westnile.ca.gov/resources_reports.php?resource_category_id=9 [Last accessed 24 Nov 2021].

6. Fleige S, Pfaffl MW. RNA integrity and the effect on the real-time qRT-PCR performance. Mol Aspects Med 2006;27:126-39. DOI PubMed

7. Baleriola C, Johal H, Jacka B, et al. Stability of hepatitis C virus, HIV, and hepatitis B virus nucleic acids in plasma samples after long-term storage at $-20^{\circ} \mathrm{C}$ and $-70^{\circ} \mathrm{C}$. J Clin Microbiol 2011;49:3163-7. DOI PubMed PMC

8. Comert F, Aktas E, Terzi HA, et al. Evaluation of hepatitis C virus RNA stability in room temperature and multiple freeze-thaw cycles by COBAS AmpliPrep/COBAS TaqMan HCV. Diagn Microbiol Infect Dis 2013;75:81-5. DOI PubMed

9. Huang LH, Lin PH, Tsai KW, et al. The effects of storage temperature and duration of blood samples on DNA and RNA qualities. PLoS One 2017;12:e0184692. DOI PubMed PMC

10. Baust JM. Biopreservation: the impact of freezing and cold storage on sample quality. Available from: https://www.eppendorf.com/product-media/doc/en/156413/Eppendorf_Freezers_White-Paper_031_EppendorfFreezer_Biopreservation-Impact-Freezing-Cold-Storage-Sample-Quality.pdf [Last accessed 24 Nov 2021].

11. Erandio J, Grippin C, Nakata M, Bear-Johnson M. Effects of storage temperature and freeze-thaw cycles on the stability of West Nile virus positive mosquito homogenate in the Ambion(R) MagMAXTM lysis/binding solution. Proceedings and papers of the 88th annual conference of the Mosquito and Vector Control Association of California; 2020 Oct; Sacramento, California. California: Mosquito and Vector Control Association of California; 2020.

12. Brault AC, Fang Y, Reisen WK. Multiplex qRT-PCR for the detection of western equine encephalomyelitis, St. Louis encephalitis, and West Nile viral RNA in mosquito pools (Diptera: Culicidae). J Med Entomol 2015;52:491-9. DOI PubMed PMC

13. Stephenson JR, Warnes A. Diagnostic virology protocols. Methods in molecular MedicineTM. Totowa, New Jersey: Humana Press; 1998. p.12.

14. Watzinger F, Suda M, Preuner S, et al. Real-time quantitative PCR assays for detection and monitoring of pathogenic human viruses in 
immunosuppressed pediatric patients. J Clin Microbiol 2004;42:5189-98. DOI PubMed PMC

15. Merrill DR, Wade CD, Fahnestock P, Baker RO. Long-term and short-term stability of viruses depend on storage temperature and preservation method. BEI Resources 2012. DOI

16. Burkhalter KL, Savage HM. Detection of Zika virus in desiccated mosquitoes by real-time reverse transcription PCR and plaque assay. Emerg Infect Dis 2017;23:680-1. DOI PubMed PMC

17. Liu H, Gan Y, Wu Y, Weng H, Lei P, Shen G. Effects of different lysis buffers of nucleic acid purification kit on the stability of influenza virus RNA. Future Virology 2014;9:549-55. DOI

18. Mcgann LE, Yang H, Walterson M. Manifestations of cell damage after freezing and thawing. Cryobiology 1988;25:178-85. DOI PubMed

19. Holanda Duarte NF, Alencar CH, Pires Neto RDJ, et al. Integration of human rabies surveillance and preventive measures in the State of Ceará, Northeast Brazil. One Health Implement Res 2021;1:17-30. DOI

20. Fouque F, Reeder JC. Impact of past and on-going changes on climate and weather on vector-borne diseases transmission: a look at the evidence. Infect Dis Poverty 2019;8:51. DOI PubMed PMC

21. Livak KJ, Schmittgen TD. Analysis of relative gene expression data using real-time quantitative PCR and the 2(-Delta Delta C(T)) method. Methods 2001;25:402-8. DOI PubMed

22. Turell MJ, Spring AR, Miller MK, Cannon CE. Effect of holding conditions on the detection of West Nile viral RNA by reverse transcriptase-polymerase chain reaction from mosquito (Diptera: Culicidae) pools. J Med Entomol 2002;39:1-3. DOI PubMed

23. Griffiths KR, Burke DG, Emslie KR. Quantitative polymerase chain reaction: a framework for improving the quality of results and estimating uncertainty of measurement. Anal Methods 2011;3:2201. DOI

24. Ryba S, Tacner J, Havlickova M, Stopka P. Stability of influenza virus as evaluated by integrity of its RNA. Acta Virol 2012;56:125-8. DOI PubMed

25. Andrews ES, Turell MJ. Effect of holding conditions on the detection of chikungunya and Venezuelan equine encephalitis viruses in mosquito pools. J Am Mosq Control Assoc 2016;32:51-4. DOI PubMed 